\title{
Early detection of basal stem rot disease (Ganoderma) in oil palms based on hyperspectral reflectance data using pattern recognition algorithms
}

\begin{abstract}
Basal stem rot (BSR) is a fatal fungal (Ganoderma) disease of oil palm plantations and has a significant impact on the production of palm oil in Malaysia. Because there is no effective treatment to control this disease, early detection of BSR is vital for sustainable disease management. The limitations of visual detection have led to an interest in the development of spectroscopically based detection techniques for rapid diagnosis of this disease. The aim of this work was to develop a procedure for early and accurate detection and differentiation of Ganoderma disease with different severities, based on spectral analysis and statistical models. Reflectance spectroscopy analysis ranging from the visible to near infrared region (325-1075 $\mathrm{nm}$ ) was applied to analyse oil palm leaf samples of 47 healthy (G0), 55 slightly damaged (G1), 48 moderately damaged (G2), and 40 heavily damaged (G3) trees in order to detect and quantify Ganoderma disease at different levels of severity. Reflectance spectra were preprocessed, and principal component analysis (PCA) was performed on different preprocessed datasets including the raw dataset, first derivative, and second derivative datasets. The classification models: linear and quadratic discrimination analysis, k-nearest neighbour $(\mathrm{kNN})$, and Naïve-Bayes were applied to PC scores for classifying four levels of stress in BSR-infected oil palm trees. The analysis showed that the $\mathrm{kNN}$-based model predicted the disease with a high average overall classification accuracy of $97 \%$ with the second derivative dataset. Results confirmed the usefulness and efficiency of the spectrally based classification approach in rapid screening of BSR in oil palm.
\end{abstract}

Keyword: Basal stem rot disease (Ganoderma); Oil palm plantations 